\title{
LA GUERRA COMERCIAL ENTRE ESTADOS UNIDOS Y CHINA: UN ENFRENTAMIENTO MÁS ALLÁ DE LOS ARANCELES
}

\author{
U.S.-CHINA TRADE WAR: A CLASH BEYOND TARIFFS
}

\author{
Kendall Ariana López-Peña ${ }^{1}$ \\ Roy Mora-Vega ${ }^{2}$
}

\begin{tabular}{|l|l|}
\hline Recibido: 03.03.19 & Aprobado: 2.12.19 \\
\hline
\end{tabular}

DOI: $10.15517 /$ isucr.v20i42.41853

\section{Resumen}

El artículo realiza una reflexión sobre la coyuntura en la que Estados Unidos inició una guerra comercial contra China, posicionando al comercio internacional como un instrumento de la política internacional, para obtener objetivos que van más allá de la cuestión económica. Se considera que esta situación de crisis comercial responde a un interés de posicionamiento geopolítico por parte de Estados Unidos.

Además, se afirma que esta situación representa una afrenta al orden liberal internacional, que históricamente ha sido liderado por los Estados Unidos, pero que en esta ocasión está erosionando deliberadamente sus posturas tradicionales de libre comercio; con el objetivo de afectar a su más cercano competidor internacional por la hegemonía global, representado por China.

Palabras claves: Estados Unidos; China; Guerra Comercial; orden liberal.

\begin{abstract}
The article reflects on the situation at which the United States waged a trade war against China, positioning international trade as an instrument of international policy, to achieve objectives that go beyond the issue Economic. This situation of trade crisis is considered to be in the interest of geopolitical positioning by the United States.

Moreover, it is claimed that this situation represents an affront to the international liberal order, which has historically been led by the United States, but is on this occasion eroding its traditional free trade stances; with the aim of affecting its closest international competitor by global hegemony, represented by China.
\end{abstract}

Key words: United States; China; Trade War; liberal order.

\footnotetext{
${ }^{1}$ Costarricense. Docente Universidad Nacional, Costa Rica. Email: kendall.lopez@uabc.edu.mx

${ }^{2}$ Costarricense. Docente Universidad Nacional, Costa Rica. Email:roy.mora.vega@una.ac.cr
} 
Introducción

El estudio de las Relaciones Internacionales ha evolucionado y las coyunturas son cada vez, más dinámicas y complejas, en donde nuevos actores marcan las agendas internacionales. La finalización de la II Guerra Mundial significó una de las etapas más sólidas para la gobernanza global, tras la consolidación de organismos como las Naciones Unidas y el GATT, posteriormente la Organización Mundial del Comercio. Así, países desarrollados como en desarrollo apostaron al comercio internacional como una herramienta para alcanzar el desarrollo. Sin embargo, el presente documento se fundamenta sobre dos ideas centrales; la primera de ellas consiste en que (1) "el comercio internacional no siempre es una herramienta para el desarrollo económico sino por el contrario se convierte en un instrumento de presión política" y en consecuencia, la segunda idea central se enmarca específicamente en la coyuntura en estudio de la Guerra Comercial entre China y Estados Unidos, en donde tal y como se menciona en el título de este manuscrito se basa en el argumento: (2) “una competencia geopolítica que va más allá de los aranceles”. En ese sentido, el presente artículo se estructura en tres apartados.

El primer apartado consiste en una contextualización de la guerra comercial entre China y Estados, cuyo fundamento en esta sección se establece a partir del ingreso de China al Sistema Multilateral del Comercio, lo que significa un cambio e incertidumbre en la dinámica comercial global. Asimismo, se describe el origen político de dicha guerra y se realiza una breve explicación del funcionamiento de un arancel en una economía.

El segundo apartado, consiste en analizar el argumento dos (2) de este artículo (Una competencia geopolítica que va más allá de los aranceles), en donde se explica la evolución de la política exterior de China, su postura como potencia "revisionista" y de modo particular, la importancia del rol del lenguaje (en términos de seguridad) específicamente analizado desde el enfoque Crítico de la Geopolítica y del cual ha hecho uso Estados Unidos tras el ataque del 11 de setiembre, incluso para justificar esta guerra comercial, que va más allá de la imposición de aranceles. Finalmente, un apartado de conclusiones. 
Contextualización de la guerra comercial entre China y USA

China comenzó a abrir su economía a finales de los años setenta. A comienzos de los ochenta, tomó algunas medidas para poner fin a su aislamiento, asumiendo la incorporación de la Provincia China de Taiwán al Fondo Monetario Internacional (FMI) y al Banco Mundial, de los cuales había sido uno de los miembros fundadores. En 1986 lanzó una campaña para reincorporarse al Acuerdo General sobre Tarifas y Comercio (GATT), del cual se había retirado en 1950 (Adhikari \& Yang 2002). En noviembre de 1982, el GATT otorgó a China el estatus de observador; luego participó en la Ronda de Uruguay como observador y después de 15 años de arduas negociaciones ingresa el 11 de setiembre de 2001 en donde finalmente se convierte en el miembro 143 de la OMC, bajo la motivación de que "necesita un impulso externo para superar los obstáculos internos, promover las reformas y proteger los intereses comerciales si desea mantener el rápido ritmo de crecimiento económico de los años ochenta y noventa" (Adhikari \& Yang 2002, 22). Lo anterior, marca la interacción entre China y los sistemas económicos internacionales. Habiendo entrado en una nueva etapa (Wang, et al. 2016)

Así, la incorporación del "gigante asiático" al Sistema Multilateral de Comercio, cambia la dinámica del comercio internacional y provoca cuestionamientos de sus socios comerciales acerca de los beneficios y desventajas que podría acarrear en términos de distorsiones al comercio. En ese sentido, el ingreso de China o de cualquier otro miembro a la Organización Mundial del Comercio parte de dos premisas básicas: 1) significa que el país miembro se somete (acepta) a las normas y principios de la organización y 2) representa la negociación de los aranceles (según producto) que el país aplicará para el resto de los Estados miembros, incorporándose así a la lista de desgravación arancelaria de la Organización Mundial de Comercio. De modo tal, que la OMC busca la liberación comercial a través de la regulación paulatina del comercio. Siendo los aranceles, un instrumento de política comercial que los países utilizan para minimizar y/o "controlar" el comercio, con el objetivo de proteger a la producción nacional y generar fuentes de ingreso al Estado. Sin embargo, ¿cómo particularmente funciona un arancel? Este impuesto (que puede ser fijo o ad valoren) recae sobre los productos importados y los encarece frente a los productos nacionales; siendo el consumidor quien paga el valor total $^{3}$ del bien importado; es decir un arancel se traslada automáticamente al precio de la mercancía y por lo tanto son los consumidores los principales

\footnotetext{
${ }^{3}$ Precio mundial + arancel
} 
afectados. Sus efectos se traducen en una disminución del comercio, incremento en los productos internacionales ante la disminución de competencia y una ineficiencia en la producción nacional, ya que los productores nacionales no deben competir en términos de innovación y competitividad, sino en términos de precios.

Lo anterior, resulta importante explicar para advertir la guerra comercial recientemente suscitada entre China y Estados Unidos, una guerra que además tiene un origen político, pero particularmente de percepción de seguridad por parte de Estados Unidos, en donde incluso el gobierno estadounidense en reiteradas ocasiones ha acusado al gobierno chino de la utilización de sus teléfonos para hacer espionaje, acusaciones que aún no han sido probadas.

Según el diario France24:

El Departamento de Justicia de Estados Unidos asegura que Huawei Device Co Ltd y Huawei Device USA Inc cometieron fraude electrónico y obstruyeron la justicia al robar tecnología de la empresa T-Mobile para probar la durabilidad de los Smartphone. En concreto, T-Mobile había acusado a Huawei de robar una tecnología llamada 'Tappy', que simulaba movimientos de los dedos humanos [...] La preocupación de Estados Unidos por Huawei no es nueva. A finales del 2012, un informe de la comisión de información del Congreso pedía que se le excluyera de contratos públicos junto con otra empresa china, ZTE. El reporte aseguraba que las tecnologías de estas firmas podían ser utilizadas contra la seguridad de Estados Unidos y señalaba que Huawei "no cooperó totalmente con su investigación y era reticente a explicar sus relaciones con el gobierno chino" [...] En diciembre de 2017 el Congreso volvió a advertir que la tecnología de Huawei constituía "una amenaza para la seguridad" de Estados Unidos. El 27 de marzo del 2018 el Gobierno publicó el Informe Especial de la Sección 301 sobre China, por no proteger los derechos de propiedad intelectual y negar el acceso al mercado a las innovaciones de Estados Unidos. En agosto, el presidente Donald Trump confirmó a militares y responsables del Gobierno la prohibición de utilizar equipos construidos por Huawei (France24 2019).

Datos de la BBC, argumentan que Washington aprobó una subida de aranceles del 10 al $25 \%$ a productos chinos por valor de US\$200.000 millones. El impuesto estará en vigor, en 
principio, hasta el 1 de junio del 2019. Mientras que China respondió con aranceles del 25\% a bienes estadounidenses por un valor de US\$60.000 millones, cerca de la mitad del total de las importaciones que China realiza de Estados Unidos. También está previsto que se suspendan el primero del julio de 2019. "Pero en lugar de desaparecer, todo indica que podrían aprobarse nuevos impuestos. Trump ya ha amenazado con imponer un arancel del 25\% al resto de mercancías que Estados Unidos trae de China y que representa US\$325.000 millones” (BBC 2019).

Lo anterior, evidencia una debilidad a la gobernanza de la Organización Mundial del Comercio, en primera instancia porque ningún país puede de manera antojadiza subir los aranceles y establecer medidas arancelarias de manera arbitraria a otro país miembro sin previamente demostrar afectaciones a su industria nacional, para lo cual podría ampararse bajo un instrumento de salvaguardia. El cual no es el caso de los Estados Unidos, puesto que su industria nacional (tecnología) no se encuentra viendo afectada, por el contrario, son más las implicaciones negativas de dicha alza. Así, tal incremento de aranceles por parte de ambas economías representa una violación al principio de "Nación más favorecida (NMF)"4, al atentar en contra del comercio sin discriminaciones; asimismo porque la reducción de los obstáculos al comercio es uno de los medios más evidentes de alentar el comercio. Esos obstáculos incluyen los derechos de aduana (o aranceles) y medidas tales como las prohibiciones de las importaciones o los contingentes que restringen selectivamente las cantidades importadas (OMC, 2019), de modo tal que, la imposición arbitraria y excesiva de aranceles de manera unilateral, consecuentemente significa una violación imperante al principio de "Comercio más libre: de manera gradual, mediante negociaciones" de la OMC. Es así que, al tratarse de una guerra con origen político (específicamente en términos de seguridad) y no de origen comercial, recurrir al Órgano de Resolución de Controversias de la OMC, resulta claramente innecesario (al menos para las potencias en cuestión).

Más allá de los aranceles se trata de una competencia geopolítica, que deteriora el orden liberal internacional

La guerra comercial entre China y Estados Unidos demuestra una vez más, que el comercio internacional es también un mecanismo que utilizan las potencias para influir en el Sistema

\footnotetext{
4"El trato NMF significa que cada vez que un país reduce un obstáculo al comercio o abre un mercado, tiene que hacer lo mismo para los mismos productos o servicios de todos sus interlocutores comerciales, sean ricos o pobres, débiles o fuertes". Recuperado de https://www.wto.org/spanish/thewto s/whatis s/tif s/fact2 s.htm
} 
internacional (en adelante SI). China, particularmente se ha convertido en un actor desafiante en el SI reflejando una evolución en su política exterior. El "gigante asiático" en ascenso está avanzando en el mundo, ejerciendo su influencia, y el mundo también está acomodando a China y dando forma al interior del país. Estos cambios no solo indican los ajustes bilaterales en curso y la adaptación entre China y la comunidad internacional, sino que también reflejan el desarrollo de la política exterior china y la redefinición del interés nacional (Wang et al 2016). En ese sentido, López 2018 (citando a Vélez 2018) argumenta tres etapas en la evolución de la política exterior china:

Una primera etapa marcada por una búsqueda de la independencia, la consolidación del poder y el activismo revolucionario comunista, con matiz claramente ideológico. Por otro lado, la segunda etapa de la política exterior de China tuvo un enfoque más pragmático, permeada por la "timidez de tomar el liderazgo en asuntos internacionales o de presentar una actitud que pudiera percibirse como amenazante" (Vélez 2018, p 19). Finalmente, la tercera etapa retoma el carácter nacionalista de la primera y la identidad pacífica de la segunda, destacando el objetivo de la paz en esta última etapa.

Vélez 2018 (citada por López 2018) en su artículo "De Dragón Revolucionario a Tímido y ahora rejuvenecido: las etapas de la política exterior china" expresa la existencia de una segunda etapa por parte de China, permeada de timidez y temor de proyectarse como desafiante ante asuntos internacionales. Sin embargo, dicha etapa ha quedado atrás y esta tercera fase caracterizada por el carácter nacionalista de la primera (etapa) y la identidad pacífica de la segunda (etapa), merma su matiz "pacifista" ante las provocaciones directas de Estados Unidos y de modo específico ante los factores idiosincráticos del presidente Donald Trump.

Así, en términos geopolíticos la actual guerra comercial entre China y Estados Unidos evidencia la importancia del rol del lenguaje particularmente analizado desde el enfoque Crítico de la Geopolítica y del cual ha hecho uso Estados Unidos tras el ataque del 11 de setiembre, en donde el Presidente George W. Bush basó su política exterior en la creencia de que los terroristas del Oriente Medio constituyen un oponente excepcionalmente peligroso, y lanzó lo que sería una larga guerra contra ellos (Russell 2014). De este modo, la frase "lucha contra terrorismo" toma otra connotación y se convierte en el estandarte para justificar la invasión en Irak y ahora las medidas comerciales en contra de la empresa china Huawei, así como del incremento injustificado de los aranceles a los bienes chinos que ingresen a Estados Unidos, construyendo una supuesta agenda de seguridad para 
los Estados Unidos. Esa secuencia de acontecimientos, según Russell (2014) evidencia la "profunda convicción de EE. UU. de que la marea global de eventos se ejecuta en favor de Estados Unidos (párr., 12)".

Por el mismo lado, en cuanto a las agendas geopolíticas si bien se planteaba que los Estados tomaban la exclusividad de las agendas geopolíticas, actualmente geopolíticos contemporáneos insisten en la importancia de un grupo de actores que tiene incidencia en la configuración de las agendas geopolíticas de los Estados (Bernard 2003). Este es el caso de la multinacional china, Huawei, cuyo actor constituye el parteaguas de la guerra comercial entre China y Estados Unidos; este último apoyado por la Unión Europea. Según Russell (2014) "las potencias revisionistas ${ }^{5}$ tienen agendas tan variadas y capacidades que ninguno puede proporcionar el tipo de oposición sistemática y global que hizo la Unión Soviética. Como resultado, los estadounidenses han tardado en darse cuenta de que esos Estados (China, Rusia e Irán) han socavado el orden geopolítico euroasiático en formas que complican los esfuerzos norteamericanos y europeos para construir un post-histórico, win-win mundo”. Así, ¿cómo el mundo debe ser, ¿qué y cómo será en el futuro? Surgen como fuertes cuestionamientos ante la presencia de nuevas potencias "revisionistas" (particularmente el caso de China) que han sacudido el equilibrio de poder y cambiado la dinámica de la política internacional.

Los líderes de estos países (China, Rusia e Irán) coinciden en la amenaza que representa Estados Unidos, el cual constituye el principal obstáculo para el logro de sus objetivos revisionistas. Su hostilidad hacia Washington y su orden es tanto ofensiva como defensiva: no solo esperan que la caída del poder de los Estados Unidos haga que sea más fácil reordenar sus regiones, sino que también les preocupa que Washington pueda intentar derrocarlos si la discordia dentro de sus países crece. Sin embargo, los revisionistas quieren evitar las confrontaciones directas con los Estados Unidos, excepto en circunstancias excepcionales cuando las probabilidades están fuertemente a su favor (Russell 2014, párr., 17)

Resulta evidente entonces, que en esta guerra comercial China conoce que las probabilidades están a su favor y que tras responderle al gobierno estadounidense aumenta las implicaciones negativas

\footnotetext{
${ }^{5}$ Es el término que el autor utiliza para denotar a las potencias Rusia, China e Irán, en el contexto de post Guerra Fría.
} 
para las inversiones extranjeras, las cadenas globales de valor, los precios del mercado ${ }^{6}$ y los mercados financieros ${ }^{7}$. Por lo que, los cuestionamientos antes mencionados sobre ¿cómo el mundo debe ser, ¿qué y cómo será en el futuro? Aumentan, tras la presencia de potencias que desobedecen el conjunto de principios y normas propias de la gobernanza de organismos internacionales - gobernanza global ya consolidada tras la II Guerra Mundial-. E imponen sus propias reglas del juego en materia comercial, utilizando de este modo, el comercio internacional no como herramienta para el desarrollo económico sino por el contrario como instrumento de presión política, que les permita alcanzar sus intereses nacionales y reestructurar un nuevo orden internacional que parecía ya establecido posterior a la Guerra Fría (al menos para Estados Unidos), pero particularmente donde prive ahora el método de suma-cero. Estableciendo nuevas agendas geopolíticas, de cara a una incertidumbre comercial y financiera.

Es necesario indicar que estas formas que utiliza el presidente Trump son corrosivas para la cooperación multilateral basada en normas, lo que representa un fuerte riesgo para el sistema liberal internacional que predomina desde el fin de la Segunda guerra Mundial, en el tanto, se ve deslegitimado por el que en algún momento fue su mayor propulsor. Estados Unidos está violentado los principios y compromisos más básicos del Sistema Multilateral del Comercio, del cual fue unos de sus principales artífices, frente a un estado que se ha negado sistemáticamente a ser participe del orden liberal en todas sus dimensiones, y solo ha aprovechado de manera astuta aquellos aspectos que le pueden ser de beneficios, como lo es el comercio internacional.

Esta estrategia de posicionamiento, a partir de la alteración de la política comercial, también resulta peligrosa para los mismos Estados Unidos, en el tanto su intención de generar efectos negativos

\footnotetext{
6 "Rick Helfenbein, el director ejecutivo de la Asociación Americana de Ropa y Calzado (AAFA, por sus siglas en inglés), dice que, aunque la importación de calzado desde Vietnam o Indonesia ha aumentado, el proceso de producción implica conocimientos técnicos y tecnología que no es fácilmente transferible de país a país. Helfenbein sostiene también que el $72 \%$ del calzado, el $84 \%$ de los accesorios y el $41 \%$ de toda la ropa vendida en Estados Unidos proviene del gigante asiático. "Estamos muy expuestos. No tenemos un lugar al que acudir para adquirir todos estos productos, así que estamos atrapados. Estos aranceles nos perjudicarán y será especialmente malo para el comercio minorista". BBC (2019). Recuperado de https://www.bbc.com/mundo/noticias-48336489

7 "China es uno de los principales compradores de la deuda de Estados Unidos, esto constituye una carta bajo la manga para China porque puede afectar el precio del mercado el cual ya actualmente está afectando a los mercados financieros e incluso al mercado de Soja". Video ¿Qué implicaciones tendrá la guerra comercial entre China y EEUU? Recuperado de https://actualidad.rt.com/video/315555-que-implicaciones-tendra-guerra-comercial-eeuu-china
} 
sobre China, en el largo plazo puede resultar contraproducentes ya que en el proceso ha dejado vacíos de poder que podría ser llevados por esta potencia emergente, y además ha lastimado la confianza de sus principales aliados estratégicos, tal es el caso de la Unión Europea o Canadá.

\section{Conclusiones}

El contexto económico actual de proteccionismo, liderado por potencias como Estados Unidos y consecuentemente por China (como respuesta a USA), así como la incertidumbre acerca del acceso a los mercados internacionales representan una serie de riesgos para la economía global y de modo particular, para América Latina. Los gobiernos de dicha región deben comprender la importancia de la diversificación de los mercados, cuya verdadera postura sea el libre comercio; lo cual signifique una diversificación en sus procesos de internacionalización (exportación, importación e inversión) y por tanto en las cadenas globales de valor.

Cabe destacar, además, la necesidad de que los Estados latinoamericanos se amparen en el Sistema Multilateral de Comercio. Mismo que si bien demuestra una debilidad de gobernanza tras las acciones unilaterales de potencias como Estados Unidos y China, sí representa una respuesta para los países en desarrollo de América Latina. Tal es el caso de México, que ante la amenaza de una posible imposición de aranceles por parte de los Estados Unidos, como un mecanismo de presión política ante la crisis migratoria en sus fronteras. Podría ampararse en el Órgano de Resolución de Controversias de la OMC y buscar el apoyo de la Unión Europea, quien además respalda a China en su guerra comercial con USA, tras argumentar que las acciones de Estados Unidos resultan ser un discurso que violenta un comercio libre, abierto y multilateral.

Esta guerra comercial entre China y Estados Unidos, una vez más, refleja las posturas de las teorías clásicas en el estudio de las Relaciones Internacionales - una búsqueda de sus intereses nacionales, en donde con el propósito de alcanzarlos utilizan mecanismos de presión política como el comercio internacional, pero específicamente en este caso, convierten instrumentos de política comercial como los aranceles en instrumentos de presión política, sacrificando los históricos principios liberales que ha liderado Estados Unidos en el orden internacional. 
Bibliografía

Adhikari, R \& Yang, Y. (2002) ¿Qué significará el ingreso en la OMC para China y sus socios comerciales? Recuperado de https://www.imf.org/external/pubs/ft/fandd/spa/2002/09/pdf/adhikari.pdf

BBC. (2019). La guerra comercial entre China y Estados Unidos explicada a través de unos zapatos. Recuperado de https://www.bbc.com/mundo/noticias-48336489

Bernard, S. (2003). Geopolitics of the World System. Rowman \& Littlefield Publishers, Inc.

France24. (2019). Estados Unidos vs. China: el comercio es lo de menos. Recuperado de https://www.france24.com/es/20190205-huawei-china-eeuu-competencia-geopolitica

López, A. (2018). Editorial de la Revista Relaciones Internacionales, Costa Rica. Recuperado https://revistas.una.ac.cr/index.php/ri/article/view/11637/15187

OMC. (2019). Los principios del sistema de comercio. Recuperado de https://www.wto.org/spanish/thewto_s/whatis_s/tif_s/fact2_s.htm

Russell, W. (2014). The Return of Geopolitics: The Revenge of the Revisionist Powers. Foreign Affairs 93 (3) 69-79. Recuperado de https://www.jstor.org/stable/24483407

Wang, Y., Tan, X., Ping, L., \& Fangfei, J. (2016). Sixty Years of China Foreign Affairs. China's Participation in International Organizations: Tracks and Features. Reading, United Kingdom: Paths International Ltd. 